\title{
Dynamical stress in force chains of granular media traveling on a bumpy terrain and the fragmentation of rock avalanches
}

Received: 13 January 2011 / Published online: 25 June 2011

(C) The Author(s) 2011. This article is published with open access at Springerlink.com

\begin{abstract}
The dynamics of grain fragmentation is usually ignored in the study of rapid granular flows. This is realistic for experiments on small-scale chutes or in many industrial processes where the specific granular energy is too small for particles to break but is unrealistic for natural rock avalanches, where the extremely comminuted state is perhaps the most evident aspect of the deposit. Based on observations of natural landslide deposits, it has been suggested that the stress may increase along a sequence of force chains formed by the granular material. However, simple calculations show that rock avalanche deposits exhibit a much more advanced degree of fragmentation than explainable with the static chain model. It is thus deduced that fragmentation along force chains must be combined with the effect of a bumpy topography. A simple model of a force chain moving on a rugged incline is then introduced, which exhibits increased fragmentation rate in the presence of a bumpy topography. It is shown that both the wave number and amplitude of the topographic undulations are significant in the efficiency of fragmentation.
\end{abstract}

\section{Introduction}

Landslides and rock avalanches represent a large natural laboratory for the study of granular flows [1]. Nevertheless, natural rock avalanches feature a series of properties associated to the enormous masses involved that are not reproducible in laboratory flows. The most apparent deviation is the magnitude of the apparent friction coefficient associated to the flow. While the friction coefficient measured in laboratory experiments remains constant as a function of the volume, friction appears to decrease with increasing volume of large landslides [2,3]. Rock avalanche deposits are also characterized by a dramatic degree of fragmentation [4-10], (see Fig. 1) that is absent in experimental avalanches, where the energy per unit mass is typically too low for particles to break.

Grain size distributions in natural rock avalanche deposits show a volume reduction of the grains by something between 15 and 18 orders of magnitude in volume [4]. The process of fragmentation is controlled by the dynamics of the avalanche and may in turn affect the flow and velocity of the rocky mass, as well as the structure of the final deposit. Thus, a quantitative analysis and theoretic explanation of fragment size distribution of a rock avalanche deposit at different positions and depths could provide indications on the impact energies and state of stress during the granular avalanches and offer a clue for understanding the anomalous mobility of large rock avalanches [6]. At first, fragmentation appears mostly as the result of energetic and chaotic impacts of an initially intact mass against the valley flanks, in an almost explosive fashion. However, the examination of natural deposits shows that in many cases, the order of geological structures (e.g., strata or magma intrusions) remains unaltered during the flow, signifying that not much internal displacement occurs

F. V. De Blasio $(\varangle)$

Department of Geosciences, University of Oslo,

Oslo, Norway

E-mail: fvblasio@geologi.uio.no 


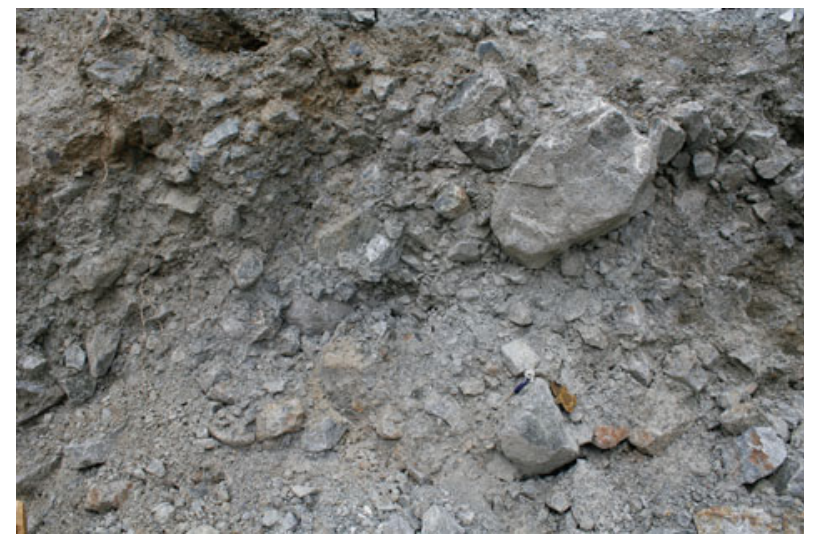

Fig. 1 Fragmented material in the deposits of the Val Pola landslide, northern Italy. Picture field about $1.5 \times 2 \mathrm{~m}$

in the body of a rock avalanche. Thus, a great deal of fragmentation probably occurs due to impulsive stresses acting vertically rather than to impact.

Despite the evident transformation of a breaking rock avalanche, the theoretic investigations of rock avalanche fragmentation have been sparse [6,7]. Measurements of the grain size spectra and their variation with the rock avalanche deposit are rarely accompanied by theoretic explanations of the observed spectra or numerical modeling of fragmentation. The present note suggests that one of the principal processes of rock avalanche fragmentation occurs through force chains in the granular medium coupled with an irregular topography for the slope path of the avalanche. A more complete model of fragmentation and the calculation of grain size distribution in a rock avalanche deposit are deferred to a next contribution.

\section{Simple estimates}

\subsection{Energy estimates}

As a starting point, we consider the energy necessary to break an intact grain of size $D$ to smaller particles of average size $d$. In the comminution industry, this is a classic problem where at least three solutions have been suggested $[11,12]$. Widely used is Bond's relation which states that the energy per unit mass necessary to disintegrate a mass from a diameter $D$ to a distribution such that $80 \%$ of the particles have diameter $<d_{80}$ is $E=10^{-2} W\left[1 / \sqrt{d_{80}}-1 / \sqrt{D}\right]$, where the Bond work index $W$ is a material constant. From Bond's equation, it is found that a slab of extremely large size $(D \rightarrow \infty)$ of a strong rock like granite will break down to particles of 3-4 mm in diameter when falling from $1000 \mathrm{~m}$ height, in the assumption that all of the gravitational energy has gone into the fragmentation channel, whereas for limestone a slightly lower value can be expected (see Appendix). The particle size predicted by the fragmentation model is compared in Table 1 with data from two landslide deposits observed in central Caucasus (Strom 2004, private communication, and ref. [13]). The evident discrepancy between observed and predicted final grain size shows, unsurprisingly, that only a small fraction of the available energy goes into the comminution process and that the fragmentation in a rock avalanche might be dynamically different from that occurring in a comminution device. Similar conclusions have been drawn by Crosta et al. [4] for the recent Valpola landslide in northern Italy. In any case,

Table 1 Comparison of prediction based on energy estimates with data (data in the fourth column extracted from observations kindly provided by Strom).

\begin{tabular}{lllll}
\hline Name of rock avalanche & Rock and bond index $(\mathrm{J} / \mathrm{kg})$ & Height of fall $(\mathrm{m})$ & $d_{80}($ observed $)(\mathrm{mm})$ & $d_{80}(\mathrm{predicted})(\mathrm{mm})$ \\
\hline Upper Mizur (1) & Limestone $(50,492)$ & 1,350 & 13.72 & 1.45 \\
Upper Mizur (2) & Limestone $(50,492)$ & 1,350 & 13.72 & 1.45 \\
Karivoh & Limestone $(50,492)$ & 2,500 & 5.66 & 0.42 \\
Karivoh (2) & Limestone $(50,492)$ & 2,500 & 5.68 & 0.42 \\
Kokomeren & Granite $(59,965)$ & 2,500 & 2.26 & 0.60 \\
\hline
\end{tabular}


it is concluded that the energy estimates are not informative on the physics of fragmentation, and one has to consider the kinetics of the fragmentation process.

\subsection{Fragmentation rates due to particle-particle impact at a certain granular temperature}

As a first model, we can consider the granular medium inside a rock avalanche as a thermalized ensemble of grains; inter-granular impacts are then due to thermal-like fluctuations in the particle speeds. A simple estimate of the fragmentation rate due to particle-particle impact can be introduced considering a system of identical grains at a certain granular temperature [14]. Without energy input, the granular temperature approximately decreases in time following Haff's law as $\mathrm{d} T / \mathrm{d} t \approx-k\left(1-\varepsilon^{2}\right) T v$ where $v=\bar{v} / s=\sqrt{2 T} / s$ is the collision frequency between neighbor particles, $k \approx 1$ is a dimensionless constant, $s$ is the average distance between the surfaces of two neighboring particles. The maximum theoretic energy input per unit time is of the order $\approx g U \sin \beta$ where $U$ is the velocity of translation of the granular flow in the gravity field of intensity $g$ and $\beta$ is the slope angle. In a stationary regime in which the energy input equates energy dissipation, the mean square velocity cannot be greater than $\bar{v}_{\mathrm{MAX}} \approx\left[g U \sin \beta s /\left(1-\varepsilon^{2}\right)\right]^{1 / 3}$, which shows the relationship between maximum velocity and average particle distance $s$. Numerous field observations show that in many levels of the deposit of a large rock avalanche, the broken grains tend to remain in relative position during their travel as pieces of a jigsaw puzzle [15]. This implies that the average distance between grains must be substantially shorter than the grain size, else grains would diffuse through the interstices. Thus, we must have $s<<R$. Considering for sake of example particles in the centimeters size range, $s \approx 1 \mathrm{~cm}$ and with plausible values $U=30 \mathrm{~m} / \mathrm{s} ; \varepsilon=0.5 ; \sin \beta=0.5$, one finds $\bar{v}<<\bar{v}_{\mathrm{MAX}}=1-2 \mathrm{~m} / \mathrm{s}$.

The specific fracture energy in particle-particle impact $e_{f}$ is defined as the kinetic energy per unit mass at which the breakage probability is $0.5,[12]$. For limestone, $e_{f} \approx 114 \mathrm{~J} / \mathrm{Kg}$, which corresponds to a relative impact velocity of $10-11 \mathrm{~m} / \mathrm{s}$, significantly greater than the maximum impact velocities estimated earlier. For smaller energies, the breakage probability $P(e)$ is found experimentally as [12] $P(e)=$ $(1 / 2)\{1+\operatorname{erf}[\ln x]\} \approx(1 / 2) \exp (-x) /(x \sqrt{\pi})$ where $x=\left(1 / \sigma_{e}\right) \ln \left(e / e_{f}\right), e$ is the specific energy (energy per unit mass), $\operatorname{erf}(x)$ is the error function, $\sigma_{e}$ is a material parameter giving the width of the distribution. As a numerical example, the data show that irrespective of rock type, the breakage probability is reduced to only 0.01 at one third of the specific fracture energy [12]. According to these estimates, the relative velocities inside a rock avalanche appear too low for particle-particle impact to be an efficient mode of fragmentation, even though impacts may cause some degree of erosion at the grain surfaces and chipping of small chunks [12]. Particle-particle collision might become more efficient at the beginning of the rock failure, when very high relative grain speeds may be present [7].

\subsection{Role of static force chains in the process of fragmentation}

Experiments and numerical modeling show that the stress in a compressed granular medium is arranged in force chains (see e.g., [16]). A grain in a force chain is subjected to more intense stress than average and is thus more likely to break. For external pressures exceeding some MPa, the distribution function of the stress acting on the grains $f$ is compatible with an exponential function $P_{1}(f)=\langle f\rangle^{-1} \exp [-f /\langle f\rangle]$; however, the distribution changes toward a Gaussian for higher pressures [17], $P_{2}(f)=\langle f\rangle^{-1} \exp \left[-(f /\langle f\rangle-1)^{2}\right]$.

Assuming a monosized population of grains with radius $R_{0}$, the maximum stress $\sigma_{f}$ that a spherical particle can withstand before breakage is assumed of the form $\sigma_{f}=\chi R^{b}$ [18], where $\chi$ and $b<0$ are material constants. Adopting the exponential distribution $P_{1}$, we can expect that if static force chains are involved in the fragmentation, then the distribution of forces would be reflected in the grain spectrum. The fraction of grains that are expected to break when subjected to a given load $P$ is then $F_{N}(R) \approx \exp \left[-\chi R^{b} / 2 P\right]$. To compare the predictions of this simple model with data, it is necessary to pass from integral spectra with respect to particle number $F_{N}(R)$ (representing the number fraction of grains of radius smaller than $R$ ) to integral spectrum with respect to volume $F_{V}(R)$, which is the fraction of volume due to grains smaller than $R$. This is because spectra are usually measured with sieve analysis, which provides measurement of volumes rather than grain numbers. Using the above equation, the integral spectrum $F_{V}(R)$ can be calculated as the ratio 
Table 2 Two different depth ranges for the deposits, corresponding pressure, observed value of $R_{50}$, observed grain radius

\begin{tabular}{lllll}
\hline Depth $(\mathrm{m})$ & Pressure $(\mathrm{MPa})$ & $R_{0}(\mathrm{~mm})$ & $R_{50}(\mathrm{obs})$ & $R_{50}($ theory $)$ \\
\hline $10-15$ & $\approx 0.2$ & 100 & $0.7-20$ & 82 \\
$60-70$ & $\approx 1.2$ & 100 & $0.3-20$ & 78 \\
\hline
\end{tabular}

The grain radius $R_{50}$ is the particle radius such that half of the deposit mass is less than that radius. Data are taken from ref. [4]

$$
F_{V}(R)=\left[\int_{0}^{R} R^{b+2} e^{-\Gamma R^{b}} d R\right]\left[\int_{0}^{R_{0}} R^{b+2} e^{-\Gamma R^{b}} d R\right]^{-1}
$$

where $\Gamma=\chi / 2 P$ and $R_{0}$ is the maximum block size. The expression can also be transformed as the ratio of two incomplete gamma functions.

Table 2 shows the results of the calculation compared to data taken from Crosta et al. [4].

According to the measurements of ref. [4], at a depth of 60-70 m, the median value of diameter distribution $D_{50}$ ranges between 0.6 and $15 \mathrm{~mm}$ depending on the position in the rock avalanche deposit. The above coupled formulas show that at these depths, particles of diameter smaller than $70 \mathrm{~mm}$ will not be broken by static force chains.

Real deposits exhibits much smaller grain sizes than predicted by the static chains model, suggesting that static chains are insufficient to cause the observed fragmentation. The two possible explanations are that: (i) the above analysis neglects the possibility of chipping, i.e., superficial erosion of grains during impact rather than grain breakage as a whole, and (ii) the fact that dynamic processes are more important than the static ones in the process of breakage along force chains. This second possibility will be explored further.

The fragmentation at slow rate along quasi-static force chains is probably more typical for basal tills, i.e., deposits underneath a glacier [19]. Such deposits are coarse-grained compared to a rock avalanche deposit.

\section{Dynamic fragmentation: force chains on a bumpy topography}

Because a rapid granular flow is traveling at high speed, the stress may augment enormously along a force chain whenever the material slides on a bumpy slope path. In the following, it is assumed that force chains are not restricted to static granular media but form continuously within a rock avalanche. Based on this premise, a simple model for the fragmentation along a single force chain is explored. To study dynamically the behavior of a force chain in a bumpy topography, we consider a simple model of a pile of $N$ spherical particles of radius $\mathrm{R}$ representing a force chain (Fig. 2). The spheres collide along the vertical direction with neighbors and dissipate energy upon each collision. The equations of motion for each particle along the direction perpendicular to the terrain are written as

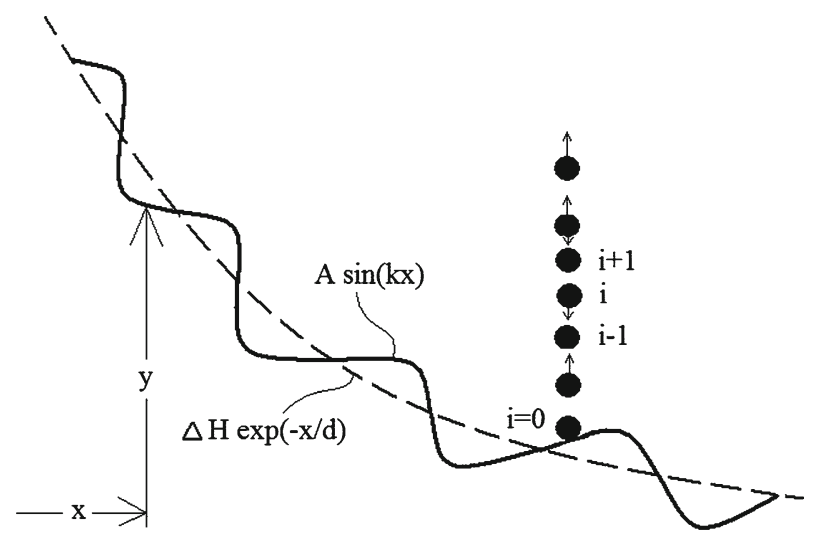

Fig. 2 Model adopted in the paper to study the fragmentation along a force chain in a granular medium 


$$
\frac{\mathrm{d} v_{i}}{\mathrm{~d} t}=-g \cos \beta-\frac{k_{\mathrm{HOOKE}}}{m}\left(\Delta_{i, i+1}+\Delta_{i, i-1}\right)-\frac{C}{m}\left(2 \dot{y}_{i}-\dot{y}_{i+1}-\dot{y}_{i-1}\right)
$$

for $i=1 \ldots N$, where $k_{\text {HOOKE }}$ is the Hooke repulsion between particles, $\Delta_{i, i+1}=\left(y_{i+1}-y_{i}-2 R\right) \Theta$ $\left(-y_{i+1}+y_{i}+2 R\right)$ is the superposition length between two neighboring particles $(\Theta(\ldots)$ is the Heaviside function), $y_{i}, m_{i}$ are the (vertical) coordinate of the $i$-th particle counted from the bottom and the mass respectively, and $C=-\ln \varepsilon\left[k_{\mathrm{HOOKE}} m /\left(\pi^{2}+\ln ^{2} \varepsilon\right)\right]$ is a constant of dissipation related to the coefficient of restitution for the velocity $\varepsilon$ [20]. The terrain topography is assigned with a parametric equation for the height $y(x)$ where $\mathrm{x}$ is the horizontal distance. The lowermost grain (with assigned index $i=0$ ) slides on the terrain with a local acceleration parallel to slope of the form $a_{\|}=g(\sin \beta-\mu \cos \beta)$ where $\beta$ is the slope angle and $\mu$ is an internal friction coefficient giving the resistance to sliding along the path. This represents the equation of motion for frictional materials with constant friction coefficient $\mu$.

The equation of motion of the other particles follows solving Eq. (2) where the position of the $i=0$ particle enters as a time-dependent boundary condition. In the absence of bumps in the topography, the angle $\beta$ changes with the position in the form of a decreasing exponential (dashed line in Fig. 1). To introduce a bumpy topography, simple sinusoidal fast-changing perturbations are superimposed to the basic exponential slope path, so that the complete slope height has the form $y(x)=\Delta H \exp (-x / d)+A \sin (k x)$ where $k$ gives the rapidity of change of the bumps height. Formally, $k$ is akin to a wave number in wave phenomena while $\lambda=2 \pi / k$ is the wave length. The horizontal motion on the rugged slope path thus determines a vertical impulse to the chain. Formally, the solution of the equations of motion is similar to that of a static chain on a vertically vibrated plate [21,22]. Because in order to estimate the fragmentation rate the present work focuses on the compressive stress between particles, the solution scheme adopted here must be necessarily based on a soft model rather than on a hard sphere model as in ref. [22]. Models for granular dissipation have been suggested where the coefficient of restitution $\varepsilon$ is linked to the properties of the material and thus depends on the impact energy [14]. The schematic nature of the present model and the fact that the velocity variations are not dramatic, justify the use of a fixed coefficient of restitution $\varepsilon$. Only one single pile is considered in the model, which is equivalent to neglect interactions between different piles.

Figure 3 shows the results of a simulation with 300 identical particles, each of $4 \mathrm{~cm}$ in diameter (for better clarity only the trajectories of the lowermost 100 particles are displayed). Panel A shows the vertical position of the grains as a function of the horizontal coordinate along the artificial slope, and panel B the separation between particles (the long-range exponential decrease is eliminated from the figure). At the beginning of the simulated sliding, the velocity parallel to bed $u_{\|}$(shown in panel C) is relatively low so that particles in the

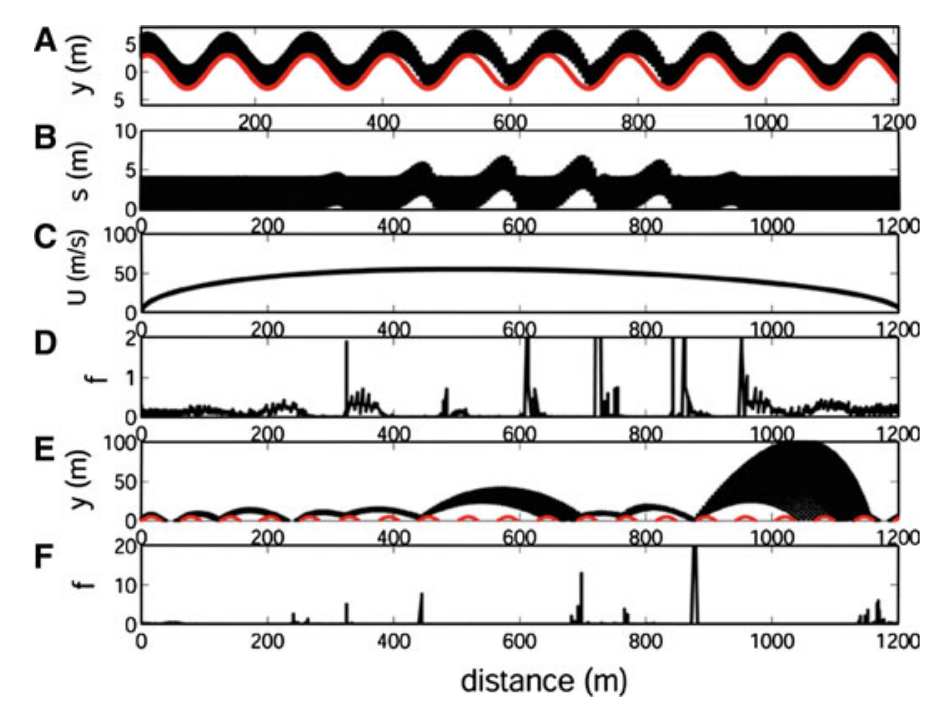

Fig. 3 Solutions of the model where the force chains is idealized as a pile of $N$ spherical particles. a Position of the lowermost 100 grains with a wave length of the topographic disturbance of $130 \mathrm{~m}$ and amplitude $2 \mathrm{~m}$. The topography is shown in red (for clarity the superimposed exponential decrease in the height is not shown). b Vertical separation s between grains. c Horizontal velocity of the chain. $\mathbf{d}$ Increase of the breakage probability per unit time with respect to the case of static chain. e Position of the grains with a stronger topographic disturbance (wave length $60 \mathrm{~m}$ and amplitude $2 \mathrm{~m}$ ). $\mathbf{f}$ Increase of the breakage probability with the topography as in $\mathbf{e}$. The numerical values used in the calculation are: $\mu=0.7, \varepsilon=0.5, k_{\mathrm{HOOKE}}=2 \times 10^{7} \mathrm{Nm}^{-1}$, $\Gamma=5.6, b=-0.343, R=2 \mathrm{~cm}, M=0.1 \mathrm{~kg}$ 
pile remain close. However, as the pile acquires speed, grains begin to collide more energetically and start to jump, as noticeable in panels A and B.

To estimate the breakage probability of grains along a pile, first the compressive stress through each grain is calculated as $\tau_{i}=k_{\text {HOOKE }}\left(\left|\Delta_{i, i+1}\right|+\left|\Delta_{i, i-1}\right|\right) /(2 R)^{2}$ [18]. This quantity is compared to the stress necessary for grain breakage $p$, which can be usefully parameterized in the form $\log _{10} p=\Gamma+(2+b) \log _{10} R$ where $\Gamma$ and $\mathrm{b}$ are material constants [18]. Panel D shows the ratio $f=\left\langle f_{i}\right\rangle=\left\langle\tau_{i} / p\right\rangle$, where the brackets denote an average over all particles. Because much fragmentation is expected to occur when $f_{i}>1$, this ratio can be considered as a proxy for fragmentation rate. It is apparently the turnover of: (1) inactive periods, (2) long-lasting bursts of high stress, and (3) episodic, very high peaks (reaching up to $f \approx 10$ ). Comparing panels $\mathrm{D}$ and $\mathrm{A}$, one can notice that quiet periods are associated with the motion of the force chain on the maxima of the topographic profile, whereas long-lasting bursts fall in the minima of the topography where an acceleration $\approx A u_{\|}^{2} k^{2}$ sums up to gravity. This latter term is due to the curvature of the bumps and represents a centrifugal acceleration term. The highest peaks are related to the collisions of the chain with the bottom following a heap, when the instantaneous compressive stress becomes very large. This calculation shows a clear correlation between breakage locations and the topographic bumps.

In panels $\mathrm{E}$ and $\mathrm{F}$, the amplitude and wave number of the topography have been accentuated, which results in much longer jumps and energetic collisions. As a rule, the more extreme the topographic features, the larger the stress peaks can potentially become. However, as the comparison between D and F shows, when the topography changes faster the heaps increase in length making the episodic bursts of fragmentation rarer (notice that the vertical scale in $\mathrm{F}$ is ten times greater than in $\mathrm{D}$ ). The threshold condition for jumping is that the dimensionless number $F=A u_{\|}^{2} k^{2} / g>1$. Thus we can conclude that an intermediate topographic disturbance produces more continuous fragmentation, whereas a fastly varying topography determines rarer and restricted locations where the fragmentation rate peaks to extreme values.

To examine more systematically the topographic effect of the fragmentation rate, Fig. 4 shows the calculations for different values of the wave number $k$ and amplitude $A$. Panel A shows the average particle separation between neighbors averaged over the simulation time. Whereas for small amplitudes and wave numbers the separation is a very small fraction of the particle diameter, when both amplitude and wave number are high (greater than $2 \mathrm{~m}$ and $0.05 \mathrm{~m}^{-1}$ respectively) the particles are cast in ballistic flights at high speed so that their separation distance reaches up to ten times the diameter. Panel B shows the parameter $L$ defined as the value of
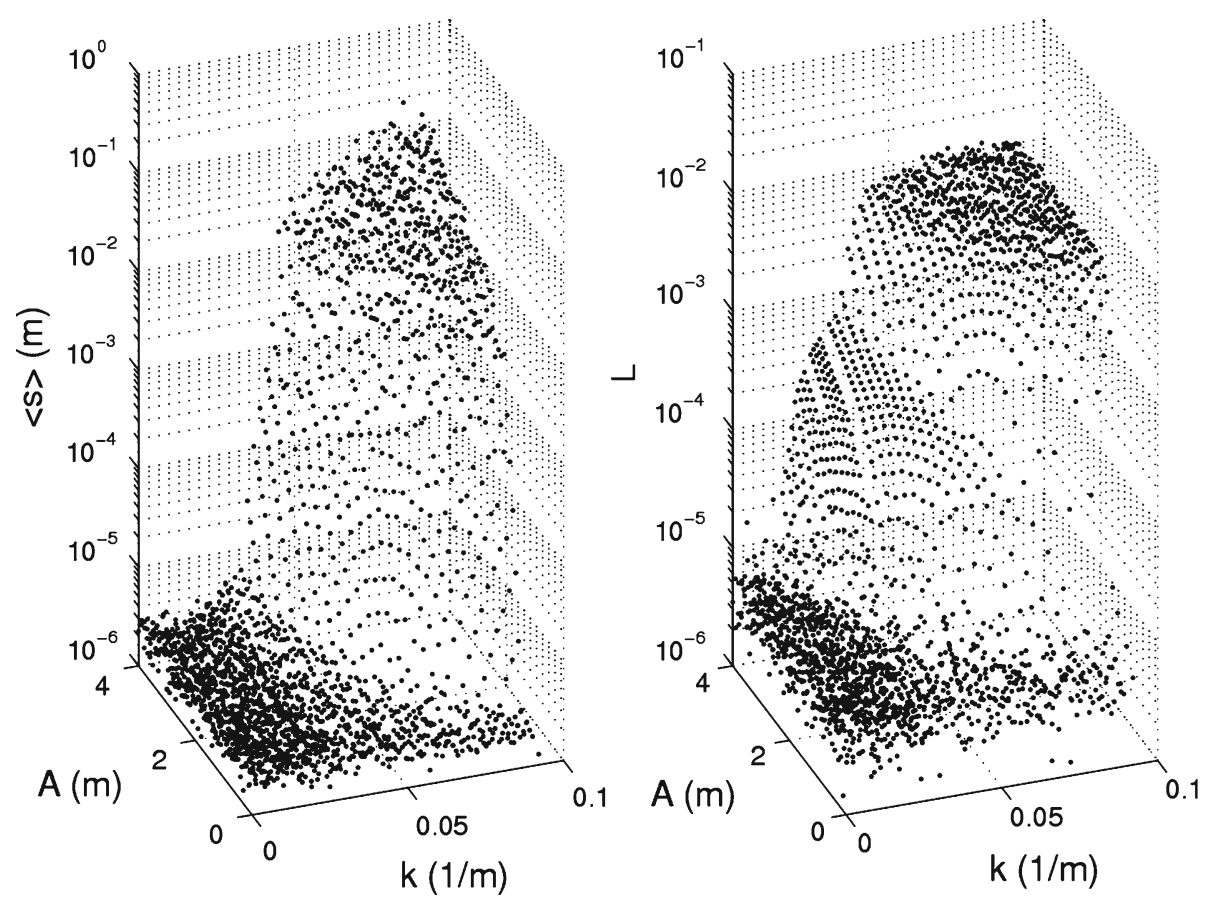

Fig. 4 Results with changing values of the topographic disturbance: amplitude A and wave number $k$, each point reporting the results of a simulation. Left, the average distance between the surfaces of neighboring particles. Right, the increase in breakage probability 
$f$ averaged over time. It appears that fragmentation increases with amplitude of the bumps and wave number. However, the figure also indicates that the fragmentation saturates when amplitude and wave number become large. This is because even though impacts are more energetic, they also become rarer. This implies that a very rapidly changing landscape will not necessarily entail higher fragmentation rate.

\title{
4 Conclusions
}

Naturally-forming force chains in a granular medium are capable of focalizing stress concentration and are more significant than particle-particle impact in bulk fragmentation of a landslide. Particles belonging to force chains are subject to major fragmentation, but only in conjunction with the sliding on an irregular terrain. Fragmentation along static chains is found to be insufficient to produce the observed clast distribution in a rock avalanche. It is then suggested that the effect of the topography is of basic importance for natural fragmentation in landslides. Fragmentation efficiency is found to increase with both amplitude and wave number of the topographic bumps, reaching limit behavior.

To conclude, considering the obvious disintegrated state of landslide bodies, it is surprising that no models have been developed for landslide fragmentation. In this preliminary paper, no attempt was made to systematize the effect of fragmentation and calculate the fragment size spectrum. Calculations with a different model of landslide fragmentation based on the kinetic of the processes and the comparison with observed spectra are deferred to another paper [23].

\begin{abstract}
Acknowledgments I am indebted with A. Strom for providing his data of rock avalanche fragmentation, at that time partly unpublished.

Open Access This article is distributed under the terms of the Creative Commons Attribution Noncommercial License which permits any noncommercial use, distribution, and reproduction in any medium, provided the original author(s) and source are credited.
\end{abstract}

\section{Appendix: comminution energies for rocks}

The calculation of the energy $d E$ required for grain size reduction $d R$ of a unit mass of rock has been addressed many times owing to the importance in the industry of comminution. The most used relationships can be summarized with the equation [11,12] $\mathrm{d} E / \mathrm{d} R=-k R^{-n}$ where $k$ and $n$ are constants. In the calculations, $n$ assumes the values $n=1$ (Kick law) $n=1.5$ (Bond law), or $n=2$ (Rittinger law). Physically, Kick's law implies that the fragmentation energy depends only on the ratio of the initial to final grain size (i.e., the energy necessary to reduce blocks of 1 meter to grains of $1 \mathrm{~cm}$ is the same as the one needed to fragment from $1 \mathrm{~cm}$ to $0.1 \mathrm{~mm}$ size), which is not realistic for rocky materials. Rittinger's law is based on the premise that the fragmentation energy is proportional to the area created with fragmentation. Bond's law does not have a direct interpretation, yet it is probably the most reliable among the simple formulas in estimates of fragmentation rates for industrial mills and crushers. The solution to the above differential equation is $E=k \ln (D / d)$ for Kick's law and $E=[k /(n-1)]\left[1 / d^{n-1}-1 / D^{n-1}\right] \approx[k /(n-1)] 1 / d^{n-1}$ for Bond and Rittinger laws. Using Bond's law, it is found that the predicted size of final particles when the rock falls for a height of $H$ meters in the gravity field is approximately $d_{80}(\mathrm{~cm}) \approx 1.04 \times 10^{-4}(W / H)^{2}$, where $W$ is the Bond work index. For example, with appropriate value for limestone $\left(W \approx 5 \times 10^{4} \mathrm{~J} \mathrm{Kg}^{-1}\right)$ one finds $d(\mathrm{~cm}) \approx 2.5 \times 10^{5} / H^{2}$ which for a $1 \mathrm{~km}$ fall yields $\approx 2.5 \mathrm{~mm}$ for the average particle diameter. The fact that the observed values of $d_{80}$ are greater shows that of the energy available only a small fraction is used up in the fragmentation of the rock avalanche.

\section{References}

1. Erismann, T.H., Abele, G.: Dynamics of Rockslides and Rockfalls. Springer, Berlin (2001)

2. Scheidegger, A.E.: On the prediction of the release and velocity of catastrophic rockfalls. Rock Mech. 5, 231-236 (1973)

3. Davies, T.R.H., McSaveney, M.J.: Runout of dry granular avalanches. Can. Geotech. J. 36, 313-320 (1999)

4. Crosta, G.B., Frattini, P., Fusi, N.: Fragmentation in the Val Pola rock avalanche, Italian Alps. JGR 112 (2007). doi:10.1029/2005JF000455

5. Locat, P., Couture, R., Leroueil, S., Locat, J., Jaboyedoff, M.: Fragmentation energy in rock avalanches. Can. Geotech. J. 43, 830-851 (2006) 
6. Davis, T.R., McSaveney, M.: The role of rock fragmentation in the motion of large landslides. Eng. Geol. 109, 67-79 (2009)

7. De Blasio, F.V.: Landslides and avalanches. In: Senneset K., Flaate K., Larsen J.O. (eds.) Taylor and Francis, London, pp. 95-100 (1995)

8. Davies, T.R., McSaveney, M.J., Hogdson, K.A.: A fragmentation-spreading model for long-runout rock avalanches. Canad. Geotech. J. 36, 1096 (1999)

9. Davies, T.R., McSaveney, M.J.: Dynamic simulation of the motion of fragmenting rock avalanches. Canad. Geotech. J. 39, 789 (2002)

10. Pollet, N., Schneider, J-L.M.: Dynamic disintegration processes accompanying transport of the Holocene Flims sturzstrom (Swiss Alps). Earth Planet. Sci. Lett. 221, 433 (2004)

11. Rhodes, M.: Introduction to Particle Technology. Wiley, Chichester (1998)

12. King, R.P.: Modeling and Simulation of Mineral Processing Systems. Butterworth Heinemann, Boston (2001)

13. Strom, A.L.: Rock avalanches of the Ardon River valley at the southern foot of the Rocky Range, Northern Caucasus, North Osetia. Landslides 1, 237-241 (2004)

14. Brilliantov, N.V., Poschel, T.: Velocity distribution in granular gases of viscoelastic particles. Phys. Rev. E 61, 2809 (2000)

15. Shreve, R.L.: The Blackhawk landslide. Geol. Soc. Amer. Spec. Paper 108, 47 (1968)

16. Mueth, D.M, Jaeger, H.M., Nagel, S.R.: Force distribution in a Granular Medium. Phys. Rev. E 57, 3164 (1998)

17. Makse, H.A, Johnson, D.L., Schwartz, L.M.: Packing of compressible granular materials. Phys. Rev. Lett. 84, 4160 (2000)

18. Mcdowell, G.R., Bolton, M.D.: On the mechanics of crushable aggregates. Geotechnique 48, 667 (1998)

19. Hooke, R., Iverson, N.R.: Grain-size distribution in deforming subglacial tills: role of grain fracture. Geology 23, $57(1995)$

20. Cleary, P.: Modelling comminution devices using DEM. Int. J. Num. An. Meth. Geomech. 25, 83-105 (2001)

21. Luding, S., Clement, E., Blumen, A., Rejchenbach, J., Duran, J.: Studies of columns of beads under external vibrations. Phys. Rev. E 49, 1634-1646 (1994)

22. Bernu, B., Delyon, F., Mazighi, R.: Steady states of a column of shaken inelastic beads. Phys. Rev. E. 50, 4551 (1994)

23. De Blasio, F.V., Crosta, G.: In preparation 